\title{
Assessment of impairment/disability due to occupational asthma through a multidimensional approach
}

\author{
M-R. Yacoub, K. Lavoie, G. Lacoste, S. Daigle, J. L’Archevêque, H. Ghezzo, \\ C. Lemière and J-L. Malo
}

ABSTRACT: Subjects with occupational asthma (OA) are often left with permanent sequelae after removal from exposure, and assessing their impairment/disability should utilise various tools.

The aim of the present study was to examine whether: 1) assessment of inflammation in induced sputum is relevant to impairment; and 2) use of questionnaires on quality of life and psychological factors can be useful for the evaluation of disability. In total, 40 subjects were prospectively assessed for permanent impairment/disability due to OA 2 yrs after cessation of exposure. Impairment was assessed as follows: 1) need for asthma medication; 2) asthma severity; 3) airway calibre and responsiveness; and 4) degree of inflammation in induced sputum. Disability was assessed according to quality of life and psychological distress.

There was a significant improvement in airway responsiveness and inflammation from diagnosis to the present assessment. Sputum eosinophils $\geqslant 2 \%$ and neutrophils $>60 \%$ were present in eight $(20 \%)$ and $12(30 \%)$ out of all subjects, respectively, one or the other feature being the only abnormalities in $15 \%$ of subjects. Quality of life was moderately affected and there was a prevalence of depression and anxiety close to $50 \%$.

In the assessment of subjects with occupational asthma, information on airway inflammation and psychological impacts are relevant to the assessment of impairment/disability, although these findings need further investigation.

KEYWORDS: Impairment/disability, occupational asthma, psychological stress, quality of life

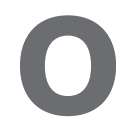
nce a diagnosis of occupational asthma $(\mathrm{OA})$ is made, the ideal course of action is to remove the worker from the work environment that is causing the OA. The individual should be considered permanently unfit to work in this environment, or in similar work environments where they would be exposed to causative agents. This prevents the deterioration of the worker's condition which would occur if the worker continued to be exposed [1,2]. Shortterm compensation and rehabilitation should be offered to enable the worker to find another job or re-train for another profession. OA can leave permanent impairment in workers even after cessation of exposure to the causal agent (see [3] for a review). The majority of subjects with OA are left with respiratory symptoms, airway obstruction, hyperresponsiveness and inflammation even years after removal from exposure to the causal agent [4] and often still require antiasthmatic medication. Bronchial hyperresponsiveness improves mostly in the first 2 yrs [5] and continues to improve thereafter, although at a slower pace [6].

Scales for assessing impairment have been proposed [3, 7, 8]. Assessment should be made when asthma status is relatively stable. Clinical criteria proposed to assess impairment include the nature and doses of medication needed to control asthma (e.g. bronchodilator, inhaled steroids), and functional criteria include levels of bronchial obstruction and hyperresponsiveness. However, these scales do not take into account residual bronchial inflammation, which can still be present after cessation of exposure. Increased levels of sputum eosinophils and neutrophils have indeed been observed in 15 and $20 \%$ of subjects, respectively, with normal airway calibre and responsiveness assessed an average of $10 \mathrm{yrs}$ after diagnosis [4].

OA can also have significant psychological and quality-of-life impacts on affected workers. To the current authors' knowledge, no studies to

\section{AFFILIATIONS}

Dept of Chest Medicine, Hôpital du Sacré-Cœur de Montréal, Montreal, QC, Canada.

\section{CORRESPONDENCE}

J-L. Malo

Dept of Chest Medicine

Hôpital du Sacré-Cœur de Montréal 5400 Gouin West Montreal

QC H4J 1 C5 Canada Fax: 15143383123 E-mail: maloj|@meddir.umontreal.ca

Received:

September 292006

Accepted after revision:

December 142006

SUPPORT STATEMENT

The present study and M-R. Yacoub's postdoctoral fellowship were funded by the Center for Asthma in the Workplace (Montreal, QC, Canada; Canadian Institutes of Health Research No. CDA66154).

STATEMENT OF INTEREST None declared.

European Respiratory Journal Print ISSN 0903-1936 Online ISSN 1399-3003 
date have evaluated the psychological impacts of OA after removal from the workplace, and few studies to date have evaluated quality of life in subjects with OA after removal from exposure $[9,10]$.

Moreover, quality of life is an important dimension of the assessment of asthma and most clinical trials assess quality of life outcomes using standardised tools [11]. Clinicians and workers' compensation agencies are requested to assess impairment that causes both functional deficits and disability. Some elements of the latter can be assessed by examining quality of life [3]. In addition, the psychological impact of being diagnosed with $\mathrm{OA}$ also needs to be assessed using relevant and standardised questionnaires.

The current authors believe that adding assessments of induced sputum and measures of quality of life and psychological distress to the evaluation of workers with OA after they are removed from exposure can significantly improve the assessment of impairment and disability in these workers. This effort can lead to designing more complete scales to assess these workers. As such, the aims of the present study were two-fold. 1) To evaluate the utility of adding assessment of airway inflammation to the standard assessment of impairment (airway calibre and responsiveness, need for medication) in subjects with OA after removal from exposure. 2) To evaluate the psychological and quality-of-life impacts of OA on subjects after removal from exposure via the administration of validated questionnaires.

\section{MATERIALS AND METHODS \\ Subjects and design}

Subjects included in the study were OA claimants who had applied to the Workers' Compensation Agency of Quebec (Commission de la santé et sécurité du travail du Québec; CSST) for assessment of impairment/disability after cessation of exposure to the agent causing OA for $\geqslant 2$ yrs. They were assessed by two out of the four Quebec CSST medical committees (the Montreal committees) that evaluate claims for occupational respiratory diseases. All claimants assessed from October 2004 to March 2006 were requested to participate on a voluntary basis by adding induced sputum, psychological and quality-of-life questionnaires to their investigation. It was made clear to the participants that assessment of impairment/ disability for current medico-legal purposes would be based only on the criteria currently used in Quebec [3], i.e. need for medication, airway calibre and responsiveness, and that the committee would not be informed of the results of the added tests, nor would these results be considered in the worker's assessment. All subjects gave written consent for their participation. The protocol was accepted by the ethics committees of both Sacré-Coeur Hospital (Montreal, Canada) and the Montreal Chest Institute, where the study took place.

\section{Tests and measures}

An open questionnaire was first used to ensure that the subject's asthma was clinically stable. Information about medication was obtained from a questionnaire administered to every subject by a trained research assistant.

Routine tests included: 1) spirometry before and after bronchodilator [12]; and 2) assessment of bronchial responsiveness to methacholine using a standardised procedure with a Wright's nebuliser (output $=0.14 \mathrm{~mL} \cdot \mathrm{min}^{-1}$; Aerosol Medical Ltd, Colchester, UK) [13]. The additional tests proposed to the participants were as follows: 1) induced sputum according to a standardised methodology [14]; 2) completion of an asthmaspecific quality-of-life questionnaire (Asthma Quality of Life Questionnaire; AQLQ) [15]; 3) completion of the St George's Asthma Severity Questionnaire [16]; and 4) completion of two psychological questionnaires, the Psychiatric Symptom Index (PSI) [17] and the Millon Clinical Multiaxial Inventory, 3rd Edition (MCMI-III) [18].

Reference values used for spirometry were those of KNUDSON et al. [19]. Normal responsiveness was set at a provocative concentration of methacholine causing a $20 \%$ fall (PC20) in forced expiratory volume in one second $(\mathrm{FEV} 1)>16 \mathrm{mg} \cdot \mathrm{mL}^{-1}$ [20]. Logarithmic transformation of PC20 was used for the statistical analysis. Changes in PC20 were considered significant when there was $a \geqslant 3$.2-fold difference from the value obtained at the time of diagnosis [21]. Different levels of sputum eosinophils were considered and significant eosinophilic airway inflammation was defined when sputum eosinophils were $\geqslant 2 \%$. The percentage of neutrophils was also assessed and values $>60 \%$ were judged to be elevated.

To assess quality of life, all patients completed the AQLQ [15]. The AQLQ includes 32 items and evaluates asthma quality of life across four life domains that may be negatively affected by asthma has follows: 1) asthma symptoms; 2) emotional dysfunction; 3) exposure to environmental stimuli; and 4) limitation of daily activities. Every field is scored from one (extremely severe) to seven (not limited at all) and the total score is the mean of the four scores. The St George's Asthma Severity Questionnaire includes eight questions related to respiratory symptoms, and the total score (range: 0-662.5) represents the sum of these elements [16]. Psychological distress was assessed using the PSI, which is a 29-item selfreport questionnaire designed to assess the presence and intensity of psychological distress in the previous 2 weeks [17]. Items are scored using a four-point scale from 0 (never) to 3 (very often). Total scores and subscale scores (depression, anxiety, anger and cognitive disturbance) are calculated as a percentage of the total possible score out of 100; scores $>25$ are considered high. Patients also completed the MCMI-III, which is a 175-item true-false inventory with 24 clinical scales designed to assess personality disturbances (e.g. avoidant personality) and psychiatric syndromes (e.g. anxiety disorders) [18]. For the purposes of the present study, only the results of the psychiatric syndrome scales will be presented. Scores $<75$ are considered within the normal range, scores 75-85 identify those with a possible psychiatric syndrome, and scores $\geqslant 85$ identify those with a high probability of a psychiatric syndrome.

\section{Analysis of results}

Functional variables such as spirometry, bronchial responsiveness and induced sputum results were compared at diagnosis and follow-up visits. Psychological and quality-of-life questionnaires were administered and examined only at the followup visit. 
Continuous variables were expressed as mean \pm SD. The other variables were expressed as median (interquartile range). Statistical analysis included unpaired t-test, ANOVA, Wilcoxon test and regression coefficients. The level of statistical significance was set at $\mathrm{p}<0.05$.

\section{RESULTS}

Selected characteristics of the participants are shown in table 1. Only five out of 45 potential subjects refused to participate (89\% participation rate). The majority were male and atopic. Although these subjects were supposed to be re-assessed 2 yrs after diagnosis according to medico-legal procedures in Quebec, the mean interval was $>3$ yrs. Low molecular weight agents were the principal agents causing $\mathrm{OA}$, and isocyanates were the leading cause in 14 subjects (35\% of all subjects included). Flour was the causal agent in five subjects, soldering and latex in four subjects each. In two subjects for whom monitoring was carried out in the workplace, the cause of OA was undetermined. A low proportion of subjects (17.5\%) were unemployed or had been employed only on a part-time basis since removal from exposure.

Results of clinical, functional and inflammatory status at the time of diagnosis and in the present study are listed in table 2 While 29 subjects required inhaled steroids either alone or combined with long-acting $\beta$-adrenergic agents at the time of diagnosis, 22 used these medications at the time of follow-up. The daily dose of inhaled steroids showed a downward trend. Whereas FEV1 and FEV1/forced vital capacity values did not significantly change, the number of subjects with slightly

\begin{tabular}{lc} 
TABLE 1 Selected baseline characteristics of participants \\
Subjects n & 40 \\
Age yrs & $46.2 \pm 11.4$ \\
Male/female & $32 / 8$ \\
Time lapse between diagnosis and assess- & $43.6 \pm 33.6$ \\
ment months & \\
Atopy\# & $29(72.5)$ \\
Smoking habit NS/ExS/S & $14 / 18 / 8$ \\
Duration of exposure to the causal agent yrs & $14.9 \pm 12.2$ \\
Duration of respiratory symptoms before & $7.0 \pm 8.7$ \\
$\quad$ leaving work yrs & \\
Nature of causal agent & \\
$\quad$ High molecular weight agents & $14(35)$ \\
Low molecular weight agents & $24(60)$ \\
Undetermined & $2(5)$ \\
Social outcome at time of assessment & \\
Work for same employer without exposure to & $8(20)$ \\
$\quad$ causal agent & \\
Work for different employer & $13(32.5)$ \\
Training for different occupation & $5(12.5)$ \\
Early retirement & $7(17.5)$ \\
Part-time job or unemployed & $7(17.5)$ \\
\hline &
\end{tabular}

Data are presented as $n$, mean \pm SD or $n(\%)$. NS: nonsmoker; ExS: ex-smoker; $\mathrm{S}$ : smoker. ${ }^{\#}$ : atopy defined by at least one immediate skin reaction to a battery of 15 ubiquitous aeroallergens; " : financial compensation is offered if needed to salary level at time of cessation of exposure to causal agent; part-time job workers were no longer exposed to the agent. increased values and the proportion of subjects with significant bronchial hyperresponsiveness ( $\mathrm{PC}_{20} \leqslant 16 \mathrm{mg} \cdot \mathrm{mL}^{-1}$ ) declined significantly. Nine $(22.5 \%)$ out of the 40 subjects showed a greater than two-fold improvement in PC20. Also, as shown in table 2, there was a significant diminution in the percentage of sputum eosinophils and significantly fewer subjects had sputum eosinophils $\geqslant 2 \%$ at the time of the current assessment. There was no significant change in sputum neutrophils. Spirometry and PC20 values at diagnosis did not differ in subjects with abnormal and normal levels of sputum eosinophils or neutrophils at follow-up.

As shown in table 3, asthma severity could be judged, on average, to be moderate, with mild-to-moderate impairments in quality of life. With the exception of the anger subscale, mean scores on all PSI subscales (depression, anxiety, cognitive disturbance), as well as the total score, were $\geqslant 25$, which is the cut-off denoting clinically significant levels of distress [17]. Moreover, more than half $(52.5 \%)$ of the subjects had scores $\geqslant 25$ on the anxiety subscale, and nearly half ( 47.5 and $45 \%)$ of the subjects had scores $\geqslant 25$ on the depression and cognitive disturbance scales, respectively, suggesting a significant level of psychological distress across multiple areas of psychological functioning. With regard to levels of psychiatric syndromes, the most common psychiatric disorder was anxiety disorders, with $14(35 \%)$ subjects having a possible $(n=5)$ or probable $(n=9)$ anxiety disorder. Levels of dysthymia (a chronic form of depression) were also high, with $22.5 \%$ of subjects having possible $(n=7)$ or probable $(n=2)$ dysthymia. Levels of all other psychiatric disturbances were $<10 \%$, and no subjects were alcohol dependent or psychotic. At follow-up, scores of all questionnaires used were not significantly different in those with and without airway hyperresponsiveness or airway inflammation. There were significant correlations $(p<0.05)$ between asthma severity on the one hand and some quality-oflife and psychological indices on the other, as follows: 1) quality of life: total score $(r=-0.44)$, symptomatic score $(r=-0.54)$, environmental score $(\mathrm{r}=-0.40)$; 2$)$ PSI: total score $(\mathrm{r}=0.36)$, anxiety $(\mathrm{r}=0.33)$, anger $(\mathrm{r}=0.33)$; and 3$)$ psychiatric syndromes (MCM1-III): somatoform disorder $(\mathrm{r}=0.33)$. No psychological scores showed significant differences according to the various social outcomes listed in table 1.

Table 4 summarises the findings in terms of specific alterations. Five $(12.5 \%)$ subjects could be considered to be cured, as all indices were within normal limits. Persistence of bronchial hyperresponsiveness was the most common abnormality, this being the only abnormality at follow-up in $22.5 \%$ of subjects and in combination with other features in $57.5 \%$ of subjects. The most common combination of abnormalities was the presence of airway obstruction and hyperresponsiveness $(15 \%)$. Six (15\%) subjects showed isolated sputum eosinophilia and/or neutrophilia and in $20(50 \%)$ subjects this abnormality was combined with other alterations. Six out of the eight subjects who had sputum eosinophilia were on inhaled steroids at the time of assessment. Four (33\%) out of the 12 subjects with sputum neutrophilia at the time of follow-up had isocyanate-induced OA, by comparison with 8 (36\%) out of 22 subjects with OA caused by other agents.

Asthma severity and daily doses of inhaled steroids correlated significantly with various functional and inflammatory indices 


\begin{tabular}{|c|c|c|c|}
\hline & Diagnosis & Current assessment & p-value \\
\hline \multicolumn{4}{|l|}{ Need for medication } \\
\hline No medication & $2(5)$ & $8(20)$ & \\
\hline Short-acting bronchodilator only & 9 (22.5) & $10(25)$ & \\
\hline Inhaled corticosteroid & $15(37.5)$ & $7(17.5)$ & \\
\hline Inhaled corticosteroid and long-acting bronchodilator & $14(35)$ & $15(37.5)$ & $\mathrm{NS}^{\#}$ \\
\hline Daily dose of inhaled corticosteroid $\mathrm{mg}$ & $609 \pm 704$ & $449 \pm 507$ & 0.06 \\
\hline FEV $_{1} \%$ pred & $81.5 \pm 18.6$ & $82.7 \pm 20.6$ & NS \\
\hline $\mathrm{FEV}_{1 / \mathrm{FVC}} \%$ & $96.7 \pm 15.4$ & $97 \pm 15.1$ & NS \\
\hline Significant bronchial hyperresponsiveness & 39 (97.5) & $22(55)$ & 0.01 \\
\hline \multicolumn{4}{|l|}{$\mathrm{PC}_{20} \mathrm{mg} \cdot \mathrm{mL}^{-1}$} \\
\hline$>16$ & 1 & 10 & \\
\hline $2-16$ & 11 & 13 & \\
\hline $0.25-<2$ & 16 & 9 & \\
\hline$<0.25$ & 12 & 0 & \\
\hline \multicolumn{4}{|l|}{ Changes in $\mathrm{PC}_{20} \mathrm{n}$-fold variation } \\
\hline$<2$ & & 24 & \\
\hline $2-<3.2$ & & 5 & \\
\hline$\geqslant 3.2$ & & 4 & \\
\hline \multicolumn{4}{|l|}{ Induced sputum } \\
\hline Total cell count $10^{6} \cdot \mathrm{mL}^{-1}$ & 1.68 (3.39) & $2.36(5.07)$ & NS \\
\hline Eosinophils & $3.5(6.2)$ & $0.5(2.0)$ & 0.005 \\
\hline \multicolumn{4}{|l|}{ Eosinophils \% } \\
\hline$<1$ & 9 & 18 & \\
\hline $1-<2$ & 3 & 8 & \\
\hline$\geqslant 2-<5$ & 9 & 2 & $0.01^{\circ}$ \\
\hline$\geqslant 5$ & 10 & 6 & $0.01^{\circ}$ \\
\hline Neutrophils & $42.1(51.0)$ & 46.5 (47.1) & NS \\
\hline Neutrophils $>60 \%$ & $10(32)$ & $12(35)$ & NS \\
\hline
\end{tabular}

such as FEV1, PC20 and sputum eosinophilia (table 5). There was no significant correlation between the levels of sputum eosinophilia or neutrophilia on the one hand and PC20 on the other.

\section{DISCUSSION}

The present study confirms that subjects with OA are often not only left with airway hyperresponsiveness, as found in most follow-up studies of OA [22], but also airway inflammation as shown by abnormal levels of eosinophils or neutrophils in induced sputum [4]. In $50 \%$ of subjects these abnormalities were present and, in $10 \%$ of subjects, this was the only abnormal finding observed at follow-up. The proportion of subjects with evidence of airway inflammation is higher than in a previous study [4] in which increased eosinophils and neutrophils were documented in only 15 and $20 \%$ of subjects, respectively. This difference can be explained by the fact that subjects of the present study were assessed 2 yrs after cessation of exposure, whereas the average cessation of exposure was nearly 10 yrs in the cited study [4].

Recent studies have examined the prognostic impact of inflammation on asthmatic flare-ups [23, 24]. The presence of increased levels of eosinophils has been consistently found to increase the risk of asthmatic flare-ups. The fact that subjects with OA have persistent increased eosinophils in induced sputum, even without airway obstruction and hyperresponsiveness (this represented $10 \%$ of subjects), should be considered when determining levels of impairment in these subjects because they are at high risk of asthma exacerbation. Moreover, airway calibre and hyperresponsiveness was not related to the level of eosinophils in induced sputum (table 5). Currently, airway calibre and responsiveness, as well as need for medication, are the only three criteria used to determine levels of impairment in scales that are proposed to assess impairment [8]. Therefore, the results of the present study point to the relevance of adding information about the status of 


\begin{tabular}{|c|c|c|}
\hline & & Score \\
\hline Asthma severity ${ }^{\#}$ & & $330 \pm 186$ \\
\hline \multicolumn{3}{|l|}{ Quality of life } \\
\hline Total score & & $4.5 \pm 1.4$ \\
\hline Respiratory symptoms & & $4.6 \pm 1.5$ \\
\hline Limitation in daily activities & & $4.1 \pm 1.3$ \\
\hline Emotional aspects & & $4.9 \pm 1.8$ \\
\hline Reactions to environmental stimuli & & $4.3 \pm 1.6$ \\
\hline \multicolumn{3}{|l|}{ Psychological distress PSI $>25$} \\
\hline Depression & $19(47.5)$ & $27.1 \pm 23.2$ \\
\hline Anxiety & $21(52.5)$ & $30.1 \pm 21.2$ \\
\hline Anger & $16(40)$ & $23.4 \pm 20.7$ \\
\hline Cognitive disturbance & $18(45)$ & $27.7 \pm 23.9$ \\
\hline Total & $19(47.5)$ & $28.1 \pm 19.9$ \\
\hline \multicolumn{3}{|l|}{ Psychiatric syndromes MCMI-III } \\
\hline \multicolumn{3}{|l|}{$<75 / 75-<85 / \geqslant 85$} \\
\hline \multicolumn{3}{|l|}{ Anxiety disorders } \\
\hline Anxiety & $26 / 5 / 9$ & $44.0 \pm 38.9$ \\
\hline PTSD & $38 / 2 / 0$ & $29.3 \pm 27.6$ \\
\hline Somatoform disorder & $38 / 1 / 1$ & $50.5 \pm 29.0$ \\
\hline \multicolumn{3}{|l|}{ Mood disorders } \\
\hline Major depression & $37 / 1 / 2$ & $44.6 \pm 27.4$ \\
\hline Dysthymia & $31 / 7 / 2$ & $35.2 \pm 32.4$ \\
\hline Bipolar disorder & $38 / 2 / 0$ & $46.0 \pm 23.3$ \\
\hline \multicolumn{3}{|l|}{ Substance abuse disorders } \\
\hline Alcohol dependence & $40 / 0 / 0$ & $24.6 \pm 23.5$ \\
\hline Drug dependence & $39 / 1 / 0$ & $34.8 \pm 20.8$ \\
\hline \multicolumn{3}{|l|}{ Severe psychotic disorders } \\
\hline Psychosis & $40 / 0 / 0$ & $31.4 \pm 29.8$ \\
\hline Delusional disorder & $39 / 0 / 1$ & $32.9 \pm 28.5$ \\
\hline
\end{tabular}

Data for the score of each test is given as mean \pm SD. For indices of the Psychiatric Symptom Index (PSI), the number of subjects with a score $>25$ is given as $n$ (\%). For indices of the Millon Clinical Multiaxial Inventory-III (MCMIIII), subjects with scores of $<75,75-<85$ and $\geqslant 85$, respectively, are given as $n$. PTSD: post-traumatic stress disorder. ${ }^{\#}$ : scale from 0 to 662.5 (severe); ": scale from 1 (bad) to 7 (excellent).

airway inflammation. Although the results should be confirmed by other studies, the current authors propose that the information obtained from induced sputum be used to quantify the degree of impairment. First, seven (17.5\%) out of 40 subjects had isolated increased sputum eosinophils $(n=1)$ or neutrophils $(n=4)$ or both eosinophils and neutrophils $(n=2$; table 4). Moreover, 13 out of the 20 subjects with airway inflammation had other abnormalities, with this combination possibly making them even more at risk of asthmatic exacerbations. Neutrophilic inflammation is a well documented phenomenon in chronic asthma, although it is not known whether this represents a prognostic factor in asthma flare-ups (although this has been shown to be the case with eosinophils) [25]. ANEES et al. [26] have shown that subjects with OA due to low molecular weight agents may show two patterns of changes in sputum while at work, either eosinophilic or noneosinophilic inflammation: subjects with eosinophilic inflammation show lower FEV1 and more pronounced airway responsiveness. Although subjects in the present study were studied, on average, $>3$ yrs after cessation of exposure, some clinical and functional parameters were significantly correlated with the levels of eosinophils and neutrophils (table 5); for instance, levels of neutrophils were inversely correlated with baseline FEV1. The current authors have also shown that subjects with $\mathrm{OA}$ due to isocyanates may show neutrophilic inflammation at the time of specific inhalation challenge [27] although, in the present study, the proportion of subjects with sputum neutrophilia was not higher in the case of OA due to isocyanates. Therefore, sputum cells may differ at the time of exposure and after cessation, although levels of eosinophils or neutrophils correlate with some clinical and functional parameters in one or other of these situations.

One study has shown that the quality of life of OA subjects 2 yrs after removal from the workplace is worse than nonoccupational asthmatics with comparable asthma severity [9]. In a recent study examining quality of life, using the same questionnaire [11] as used in the previous [9] and current studies by the present authors' group, AL-OTAiBI et al. [10] found a minimal impact in 10 asthmatic subjects with latex allergy who were assessed, on average, 2 yrs after cessation of exposure. Compared with scores reported in this previous study, quality-of-life scores were worse in subjects in the present study; the reason for this is not known. It is unlikely to be due to changes in compensation in the interval of $15 \mathrm{yrs}$ between the two studies, as the cost of compensation has increased significantly, from an average of CAN\$50,000 to CAN\$75,000 over this period in Quebec. The lower quality-oflife scores observed in the present study could be due to relatively high levels of psychological distress reported by patients, which may have affected quality-of-life levels. Previous studies have shown that asthma patients with depressive and anxiety disorders have worse asthma-related quality of life compared with patients with these disorders, independent of age, sex, asthma duration and severity [28, 29].

In the current study, levels of psychological distress were assessed using a general symptom index (PSI) and an inventory that assesses levels of psychiatric syndromes (MCMI-III). Levels of psychological distress were observed to be slightly elevated according to scores on the PSI. Levels of anxiety, depression and cognitive disturbance were all in the clinical range $(>25)$, suggesting that the psychological consequences of $\mathrm{OA}$ are not only significant but affect a range of psychological factors. Moreover, the fact that psychological distress was measured $\geqslant 2$ yrs after OA was diagnosed and 2 yrs after subjects had been removed from the workplace suggests that psychological distress persists beyond the shock of the initial diagnosis and withdrawal from the workplace. With respect to psychiatric syndromes which reflect more severe psychopathology, anxiety disorders and dysthymia were relatively common, with 35 and $22.5 \%$ of patients having a possible or probable anxiety disorder or dysthymia, respectively. This suggests that patients with OA are anxious and some are chronically depressed, a finding that is consistent with previous studies of nonoccupational asthmatics [28]. However, rates of anxiety disorders and dysthymia in the present study were much higher than those observed in the study of tertiary care asthmatics by LAVOIE et al. [28], which 


\begin{tabular}{|c|c|c|c|c|c|c|}
\hline Pattern & No abnormality & Bronchial obstruction & Hyperresponsiveness & $\begin{array}{l}\text { Sputum neutrophilia } \\
\qquad>60 \%\end{array}$ & $\begin{array}{c}\text { Sputum eosinophilia } \\
\geqslant 2 \%\end{array}$ & Subjects \\
\hline 1 & X & & & & & 5 \\
\hline 2 & & $x$ & & & & 2 \\
\hline 3 & & & $x$ & & & 9 \\
\hline 4 & & & & $x$ & & 3 \\
\hline 5 & & & & & $x$ & 1 \\
\hline 6 & & $x$ & $x$ & & & 6 \\
\hline 7 & & $x$ & & $x$ & & 4 \\
\hline 8 & & & $x$ & $x$ & & 1 \\
\hline 9 & & & $x$ & & $x$ & 3 \\
\hline 10 & & & & $x$ & $x$ & 2 \\
\hline 11 & & $x$ & $x$ & $x$ & & 2 \\
\hline 12 & & $x$ & $x$ & & $x$ & 2 \\
\hline Total & 5 & 16 & 23 & 12 & 8 & 40 \\
\hline
\end{tabular}

Data are presented as $\mathrm{n}$. X indicates the characteristics present in each outcome pattern.

reported rates of anxiety disorders at $25 \%$ and rates of dysthymia at only $4 \%$. However, the study by LAVOIE et al. [28], as well as most studies in the literature, used a psychiatric interview to assess psychiatric disorders, which may provide a more conservative estimate of rates of psychiatric disorder than self-report questionnaires, such as the ones used in the present study [30].

Disability is defined by the American Thoracic Society as the "total effect of an impairment on a patient's life" [7]. The present study confirms that disability is not generally associated with the extent of impairment. The use of answers to quality-of-life questionnaires has been advocated in examining disability due to OA [22]. The current authors believe that the use of other standardised psychological tools, such as those used in the present study, should also be advocated to provide extra information that can be used in assessing disability. These questionnaires should be used both at the time of diagnosis and follow-up so as to compare the same worker in both situations. This is a limitation of the present study, in which these tools were only used at the follow-up. Although the study was carried out in the context of assessing permanent impairment for medico-legal purposes in subjects with OA, the current authors do not believe that this could have significantly affected answers to questions. Indeed, subjects were clearly informed that the extra information obtained from these questionnaires would not be used for the assessment of impairment/disability since this was a research project.

Disability is related to the social impact of OA. In Quebec, rehabilitation programmes that are run in the first 2 yrs following removal from exposure have satisfactory efficiency, as only a minority of workers are left unemployed or hold only part-time jobs [31]. In the current study, this proportion was $17.5 \%$ compared with $8 \%$ in a previous study [31]. Therefore, other studies addressing the question of disability in situations of less satisfactory socioeconomic outcomes should be carried out. Indeed, OA and work-related asthma have significant socioeconomic impact. Unemployment affects $20-38 \%$ of

\section{TABLE 5 Relationships between clinical, functional and inflammatory parameters}

\begin{tabular}{|c|c|c|c|c|c|c|}
\hline & $\mathrm{PC}_{20}$ & Sputum eosinophilia & Sputum neutrophilia & $\begin{array}{c}\text { Daily dose of inhaled } \\
\text { steroids }\end{array}$ & Quality of life & Asthma severity \\
\hline $\mathrm{PC}_{20}$ & & $-0.28 ; 0.16$ & $0.11 ; 0.58$ & $-0.39 ; 0.027$ & $0.41 ; 0.016$ & $-0.32 ; 0.08$ \\
\hline Sputum eosinophilia & & & $-0.2 ; 0.27$ & $0.30 ; 0.009$ & $-0.15 ; 0.39$ & $0.39 ; 0.03$ \\
\hline Sputum neutrophilia & & & & $0.16 ; 0.36$ & $-0.21 ; 0.24$ & $0.27 ; 0.15$ \\
\hline Quality of life & & & & & & $-0.05 ; 0.75$ \\
\hline
\end{tabular}

Data are presented as r-value; p-value. PC20: provocative concentration causing a $20 \%$ fall in forced expiratory volume in one second (FEV1); \% pred: \% predicted. Asthma severity was assessed with the St George's Asthma Severity Questionnaire [16]; quality of life was assessed according to the questionnaire proposed by JuNIPER et al. [15]; daily dose of inhaled steroids in beclomethasone equivalent; sputum neutrophilia and eosinophilia in \%; log transformation of PC20. 
workers after diagnosis as documented in studies carried in the UK, France, Belgium and British Columbia (Canada) [32].

In conclusion, the results of the present study may contribute to the development of a scale that can more accurately assess permanent impairment due to occupational asthma, a scale based not only on physiological parameters but also on information obtained from assessment of inflammation. The current scale proposed in 1993 by the American Thoracic Society [7], which retained previously published criteria [33] has not been revised for nearly 15 yrs, although its implementation is recent [8]. In addition, the study included psychological and quality-of-life measures that provide information on psychosocial disability due to occupational asthma. Although physicians are often requested to provide advice not only on impairment but also on disability, they are generally hesitant and reluctant to commit to a judgment on the latter, probably because they are less familiar with the validated tools used to measure quality of life and psychological variables. Nonetheless, these tools are widely available and may provide valuable information with which to assess the psychosocial impacts of occupational asthma.

\section{ACKNOWLEDGEMENTS}

The authors would like to thank G. Ostiguy, L. Cicora and M-J. Provencher (Montreal, QC, Canada) for their valuable assistance and $\mathrm{K}$. Lieber for reviewing the manuscript.

\section{REFERENCES}

1 Côté J, Kennedy S, Chan-Yeung M. Outcome of patients with cedar asthma with continuous exposure. Am Rev Respir Dis 1990; 141: 373-376.

2 Moscato G, Dellabianca A, Perfetti L, et al. Occupational asthma: a longitudinal study on the clinical and socioeconomic outcome after diagnosis. Chest 1999; 115: 249-256.

3 Bersntein IL, Keskinen H, Blanc PD, Chan-Yeung M, Malo JL. Medicolegal aspects, compensation aspects, and evaluation of impairment/disability. In: Bernstein IL, Chan-Yeung M, Malo JL, Bernstein DI, eds. Asthma in the Workplace. Taylor and Francis, New York, 2006; pp. 319-351.

4 Maghni K, Lemière C, Ghezzo H, Yuquan W, Malo JL. Airway inflammation after cessation of exposure to agents causing occupational asthma. Am J Respir Crit Care Med 2004; 169: 367-372.

5 Malo JL, Cartier A, Ghezzo H, Lafrance M, McCants M, Lehrer SB. Patterns of improvement in spirometry, bronchial hyperresponsiveness, and specific IgE antibody levels after cessation of exposure in occupational asthma caused by snow-crab processing. Am Rev Respir Dis 1988; 138: 807-812.

6 Malo JL, Ghezzo H. Recovery of methacholine responsiveness after end of exposure in occupational asthma. Am J Respir Crit Care Med 2004; 169: 1304-1307.

7 Guidelines for the evaluation of impairment/disability in patients with asthma. American Thoracic Society. Medical Section of the American Lung Association. Am Rev Respir Dis 1993; 147: 1056-1061.

8 Cocchiarella L, Andersson GBJ, eds. Guides to the Evaluation of Permanent Impairment. 5th Edn. Chicago, Amercian Medical Association, 2001.
9 Malo JL, Boulet LP, Dewitte JD, et al. Quality of life of subjects with occupational asthma. J Allergy Clin Immunol 1993; 91: 1121-1127.

10 Al-Otaibi S, Tarlo SM, House R. Quality of life in patients with latex allergy. Occup Med 2005; 55: 88-92.

11 Juniper EF. Quality of life in adults and children with asthma and rhinitis. Allergy 1997; 52: 971-977.

12 Standardization of spirometry, 1994 update. American Thoracic Society. Am J Respir Crit Care Med 1995; 152: 1107-1136.

13 Cockcroft DW, Killian DN, Mellon JJA, Hargreave FE. Bronchial reactivity to inhaled histamine: a method and clinical survey. Clin Allergy 1977; 7: 235-243.

14 Pizzichini MMM, Popov TA, Efthimiadis A, et al. Spontaneous and induced sputum to measure indices of airway inflammation in asthma. Am J Respir Crit Care Med 1996; 154: 866-869.

15 Juniper EF, Guyatt GH, Epstein RS, Ferrie PJ, Jaeschke R, Hiller TK. Evaluation of impairment of health-related quality of life in asthma: development of a questionnaire for use in clinical trials. Thorax 1992; 47: 76-83.

16 Jones PW, Quirk FH, Baveystock CM. The St. George's Respiratory Questionnaire. Respir Med 1991; 85: Suppl. B, 25-31.

17 Ilfeld FW. Further validation of a psychiatric symptom index in a normal population. Psychol Rep 1976; 39: 1215-1228.

18 Millon $\mathrm{T}$, ed. Millon Multiaxial Inventory. 3rd Edn. Minneapolis, National Computer Systems, 1994.

19 Knudson RJ, Lebowitz MD, Holberg CJ, Burrows B. Changes in the normal maximal expiratory flow-volume curve with growth and aging. Am Rev Respir Dis 1983; 127: 725-734.

20 Malo JL, Pineau L, Cartier A, Martin RR. Reference values of the provocative concentrations of methacholine that cause $6 \%$ and $20 \%$ changes in forced expiratory volume in one second in a normal population. Am Rev Respir Dis 1983; 128: 8-11.

21 Dehaut P, Rachiele A, Martin RR, Malo JL. Histamine doseresponse curves in asthma: reproducibility and sensitivity of different indices to assess response. Thorax 1983; 38: 516-522.

22 Becklake MR, Chan-Yeung M, Malo JL. Epidemiological approaches in occupational asthma. In: Bernstein IL, ChanYeung M, Malo JL, Bernstein DI, eds. Asthma in the Workplace. 3rd Edn. Taylor and Francis, New York, 2006; pp. 37-85.

23 Deykin A, Lazarus SC, Fahy JV, et al. Sputum eosinophil counts predict asthma control after discontinuation of inhaled corticosteroids. J Allergy Clin Immunol 2005; 115: 720-727.

24 Belda J, Parameswaran K, Lemière C, Kamada D, O'Byrne PM, Hargreave FE. Predictors of loss of asthma control induced by corticosteroid withdrawal. Can Respir J 2006; 13: 129-133.

25 Gibson PG, Simpson JL, Saltos N. Heterogeneity of airway inflammation in persistent asthma: evidence of neutrophilic inflammation and increased sputum interleukin-8. Chest 2001; 119: 1329-1336.

26 Anees W, Huggins V, Pavord ID, Robertson AS, Burge PS. Occupational asthma due to low molecular weight agents: eosinophilic and non-eosinophilic variants. Thorax 2002; 57: $231-236$. 
27 Lemière C, Romeo P, Chaboillez S, Tremblay C, Malo JL. Airway inflammation and functional changes after exposure to different concentrations of isocyanates. J Allergy Clin Immunol 2002; 110: 641-646.

28 Lavoie KL, Cartier A, Labrecque M, et al. Are psychiatric disorders associated with worse asthma control and quality of life in asthma patients? Respir Med 2005; 99: 1249-1257.

29 Lavoie KL, Bacon SL, Barone S, Cartier A, Ditto B, Labrecque $\mathrm{M}$. What is worse for asthma control and quality of life: depressive disorders, anxiety disorders, or both? Chest 2006; 130: 1039-1047.
30 Lowe B, Spitzer RL, Grafe K, et al. Comparative validity of three screening questionnaires for DSM-IV depressive disorders and physicians' diagnoses. J Affect Disord 2004; 78: 131-140.

31 Dewitte JD, Chan-Yeung M, Malo JL. Medicolegal and compensation aspects of occupational asthma. Eur Respir J 1994; 7: 969-980.

32 Vandenplas O, Toren K, Blanc PD. Health and socioeconomic impact of work-related asthma. Eur Respir J 2003; 22: 689-697.

33 Chan-Yeung M. Occupational asthma. Chest 1990; 98: Suppl. 5, 148S-161S 